Check for updates

Cite this: RSC Adv., 2018, 8, 22617

\title{
Development of a microwave-assisted sustainable conversion of furfural hydrazones to functionalised phthalimides in ionic liquids $\dagger$
}

\author{
Valerija Karaluka, $\ddagger^{\mathrm{t}}$ Kengo Murata, $\dot{\ddagger}^{\mathrm{a}}$ Shinto Masuda, ${ }^{a}$ Yuto Shiramatsu, ${ }^{\mathrm{a}}$ \\ Takuji Kawamoto, (D) ${ }^{a}$ Helen C. Hailes, (D) *b Tom D. Sheppard (D)*b \\ and Akio Kamimura (D) *a
}

Received 7th May 2018

Accepted 11th June 2018

A sustainable synthetic procedure to convert furfural hydrazones into functionalised phthalimides was developed. The reaction was performed in a microwave using a hydrophilic ionic liquid, [bmim] [Cl], as the solvent which could be readily recovered by a simple extraction. The ionic liquid was successfully recycled with no significant loss in product yields.

performed in water and avoids the use of any organic solvent in the extraction or purification steps. However, in some cases direct crystallisation of the product from the reaction mixture did not readily take place, and purification after extraction with organic solvents was necessary. In addition, where substrates are poorly soluble in water the reaction does not proceed efficiently. In this new study, we wished to explore the development of a more general protocol applicable to more hydrophobic compounds using ionic liquids as the medium for the reaction.

Ionic liquids are recognized as new green solvents for chemical transformations, ${ }^{16-18}$ because of their low volatility, compatibility with catalysts, ability to dissolve reactants, and their unique physical properties which often enable them to catalyse organic reactions. ${ }^{16,19-21}$ Additionally, it has been reported that reactions in ionic liquids were efficiently accelerated by microwave heating because very rapid heating of IL to high temperatures was easily achieved. This advantage is mainly because of dipolar rotation and ionic conduction effects derived from the use of ionic liquids. ${ }^{22-30}$ For example, we have recently demonstrated the successful utilisation of ionic liquids for; (i) depolymerisation of plastics, ${ }^{31,32}$ (ii) cellulolysis ${ }^{33}$ using the hydrophobic IL [PP13][ $\left.\mathrm{NTf}_{2}\right]$, and (iii) conversion of sorbitol to isosorbide using [TMPA] $\left[\mathrm{NTf}_{2}\right]^{34}$

The ultimate goal of this current study is to develop a sustainable route for the conversion of biomass waste to useful aromatic compounds using ionic liquids. Herein, we report the use of an ionic liquid, [bmim][Cl], as an alternative green solvent for the synthesis of substituted phthalimides from furfurals. We also demonstrate the recyclability of [bmim] $[\mathrm{Cl}]$ in the reaction.

\section{Results and discussion}

We initially sought to identify suitable ionic liquids for the synthesis of phthalimides from furfural hydrazone $\mathbf{3 a}$ (Table 1).
${ }^{a}$ Department of Applied Chemistry, Yamaguchi University, Ube 755-8611, Japan. E-mail:ak10@yamaguchi-u.ac.jp

${ }^{b}$ Department of Chemistry, University College London, 20 Gordon Street, London, WC1H OAJ, UK

$\dagger$ Electronic supplementary information (ESI) available: Experimental procedures for the preparation of hydrazones $1 ;{ }^{1} \mathrm{H}$ and ${ }^{13} \mathrm{C}$ NMR spectra. See DOI: $10.1039 / \mathrm{c} 8 \mathrm{ra03895c}$

$\ddagger$ Authors contributed equally. 
Table 1 The Diels-Alder reaction of hydrazone 1a with maleimide 2

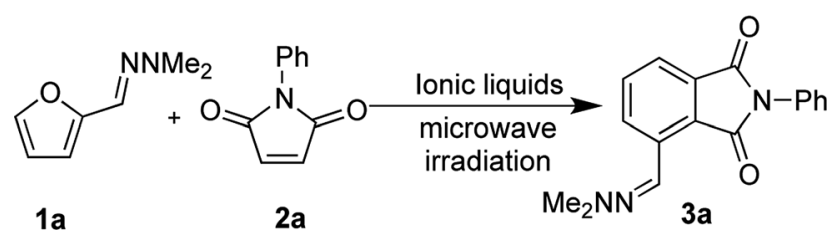

\begin{tabular}{|c|c|c|c|c|c|}
\hline Entry & Ionic liquids & Temp $\left({ }^{\circ} \mathrm{C}\right)$ & Time (min) & Yield (\%) & Recovery of IL (\%) \\
\hline 1 & {$[\mathrm{bmim}][\mathrm{Cl}]$} & 100 & 120 & $32^{a}$ & 99 \\
\hline 2 & {$[\mathrm{bmim}]\left[\mathrm{BF}_{4}\right]$} & 120 & 60 & $50^{b}$ & $46^{c}$ \\
\hline 3 & {$[\mathrm{bmim}]\left[\mathrm{NTf}_{2}\right]$} & 120 & 60 & $61^{b}$ & $97^{c}$ \\
\hline 4 & {$[\mathrm{TMPA}]\left[\mathrm{NTf}_{2}\right]$} & 100 & 120 & 57 & 88 \\
\hline 5 & {$[\mathrm{PP} 13]\left[\mathrm{NTf}_{2}\right]$} & 100 & 120 & 60 & 90 \\
\hline 6 & {$[\mathrm{DEME}]\left[\mathrm{NTf}_{2}\right]$} & 100 & 120 & 32 & 92 \\
\hline 7 & {$[\mathrm{bmim}][\mathrm{Cl}]$} & 100 & 120 & 35 & 97 \\
\hline 8 & {$[\mathrm{bmim}][\mathrm{Cl}]$} & 100 & 120 & $83^{d}$ & 99 \\
\hline
\end{tabular}

${ }^{a}$ Conventional heating was employed instead of microwave irradiation. ${ }^{b}$ Product partially contaminated with IL. ${ }^{c}$ IL partially contaminated with product 3a. ${ }^{d}$ Freshly prepared hydrazone 1a was used. When the reaction was carried out in the presence of 10 mol\% of $N, N$-dimethylhydrazine, the yield of 3a dropped to less than $10 \%$.

A variety of hydrophilic (entries 1, 2, 7, 8) and hydrophobic (entries 3-6) ionic liquids were examined. We first examined conventional heating of the reaction, but the reaction progressed slowly and the desired product $3 \mathbf{a}$ was obtained in only $32 \%$ yield (entry 1). To increase the reaction rate, microwave heating was employed. The IL $[\mathrm{bmim}]\left[\mathrm{BF}_{4}\right]$ was found to be unsuitable for the reaction due to difficulties observed in the isolation of the product (entry 2), and the low recovery of the IL. We envisaged that $[\mathrm{bmim}]\left[\mathrm{NTf}_{2}\right]$ might be effective at mediating the reaction due to its high ionicity. ${ }^{35,36}$ Although [bmim $]\left[\mathrm{NTf}_{2}\right]$ was found to give the best yield of $61 \%$ (entry 3 ), separation of the IL from the product was difficult because of its hydrophobicity, and this required copious washes with organic solvents, which was against the purpose of developing a sustainable environmentally friendly procedure. Similarly, [TMPA] $\left[\mathrm{NTf}_{2}\right]$ and [PP13][ $\left.\mathrm{NTf}_{2}\right]$ resulted in good yields of $3 \mathbf{a}$, but the recovery of the IL was not satisfactory (entries 4 and 5 , respectively). The IL [DEME] $\left[\mathrm{NTf}_{2}\right]$ was a poor solvent for the reaction with only $32 \%$ yield of the product obtained (entry 6). We then examined the hydrophilic ionic liquid $[\mathrm{bmim}][\mathrm{Cl}]$. The reaction progressed well with microwave heating, and complete separation of the IL was accomplished by washing the organic layers three times with water, although the yield of $3 \mathbf{a}$ remained at $35 \%$ (entries 7). To improve the yield of 3a, we examined the use of freshly prepared hydrazone 1a. Compound 3a was then isolated in $83 \%$ yield without contamination of the IL (entry 8). Pleasingly, $99 \%$ of the IL was recovered after the work-up. The product 3a was isolated by recrystallisation from an acetone/ water mixture without the need for chromatographic purification. It was considered that the lower yields initially observed during the formation of $\mathbf{3 a}$ could be attributed to contamination with $N, N$-dimethylaminohydrazine, liberated from hydrazone 1a on storage. Indeed, the cycloaddition reaction became sluggish when $10 \mathrm{~mol} \%$ of dimethlhydrazine was added and the yield of compound 3a decreased to less than 10\% (Table 1 footnote $d$ ). We think that contamination with small amounts of $N, N$-dimethylhydrazine in ionic liquids could progress some other reactions, possibly conjugate addition to $2 a$, preventing the formation of $\mathbf{3 a}$ in high yield.

With satisfactory reaction conditions shown in Table 1, entry 8 , we examined the applicability of the reaction conditions to a variety of furfural hydrazone derivatives $\mathbf{1}$ and maleimides $\mathbf{2}$ (Scheme 1). The reaction gave good yields of phthalimide products with aliphatic-substituted maleimides (3b-3i), including many examples of highly hydrophobic compounds that are unsuitable for reactions in water. The Diels-Alder reaction between $\mathbf{1 a}$ and $\mathbf{2 b}$ in IL gave phthalidimide $\mathbf{3 b}$ in $94 \%$ yield along with a $96 \%$ recovery of $[\mathrm{bmim}][\mathrm{Cl}]$. Longer aliphatic substituents on the maleimide were also tolerated, giving phthalimides $3 \mathbf{c}-3 \mathbf{i}$ in moderate to good yields. The reaction with a bulky maleimide such as $\mathbf{2 k}$ also progressed smoothly to give $N$-cyclohexylphthalimide $3 \mathbf{k}$ in $68 \%$ yield. Substituted benzyl maleimides similarly afforded the products $\mathbf{3 m}$ and $\mathbf{3 n}$ in excellent yields (94\% and $97 \%$, respectively). Note that the $N$ unsubstituted maleimide gave the cycloaddition product $3 \mathbf{j}$ in a good yield, which opens up the opportunity to further functionalisation at the nitrogen atom. $N$-Allyl substituted maleimide $\mathbf{2} \mathbf{l}$ underwent the cycloaddition to give the product $\mathbf{3 l}$ in $74 \%$ yield. We also examined the cycloaddition reaction using 5methylfurfural hydrazone $\mathbf{1 b}$, giving $\mathbf{3 p}$, $\mathbf{3 q}$ and $\mathbf{3 r}$ in reasonable yields.

Treatment of 5-bromofurfural hydrazone $1 \mathrm{c}$ with maleimide $2 \mathbf{a}$ and $2 \mathbf{b}$ resulted in the formation of phenol derivative $3 \mathbf{s}$ and 3t, respectively. During the formation of these compounds, elimination of hydrogen bromide instead of dehydration occurred to aromatise the adduct. Unfortunately, the yield of $\mathbf{3 t}$ remained low because of its water solubility which caused loss of the product during the extraction/washing procedure. 


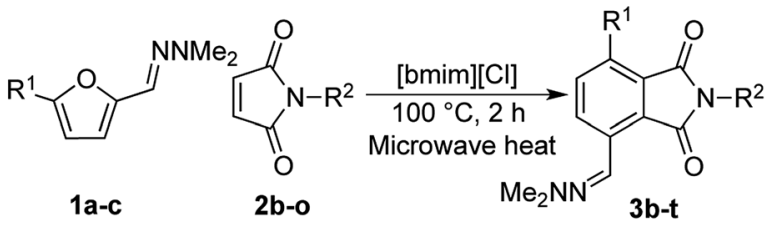<smiles>CCN1C(=O)c2cccc(CN=C(C)C)c2C1=O</smiles>

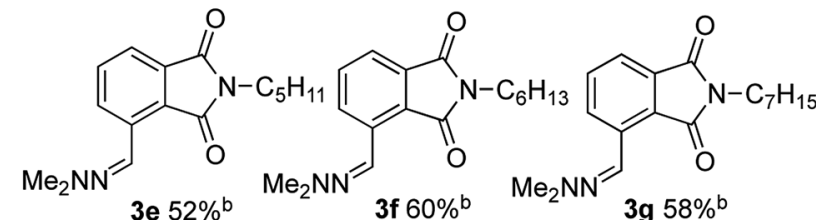<smiles>CCCN1C(=O)c2cccc(CN(C)C)c2C1=O</smiles><smiles>C=CCN1C(=O)c2cccc(CN=C(C)N)c2C1=O</smiles>

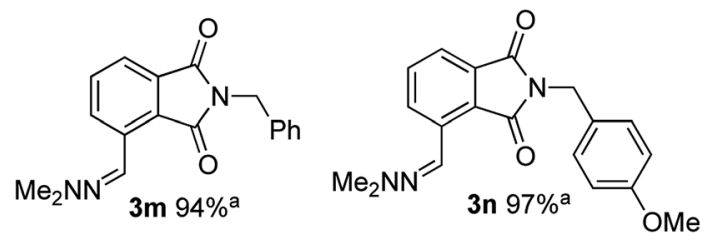<smiles>CCN1C(=O)c2c(C)ccc(C=NCc3ccc(O)c4c3C(=O)N(c3ccccc3)C4=O)c2C1=O</smiles>

Scheme 1 Cycloaddition products (3b-3t) were synthesised in a microwave reactor at $100{ }^{\circ} \mathrm{C}$ in $2 \mathrm{~h}$ with $1 \mathrm{~g}[\mathrm{bmim}][\mathrm{Cl}]$ as the solvent unless otherwise stated. The recovery of ionic liquid is $>95 \%$ unless otherwise stated; ${ }^{a} 1 \mathrm{~g}$ of IL used for $0.7 \mathrm{mmol}$ of hydrazone and $1.5 \mathrm{eq}$. maleimide; ${ }^{\mathrm{b}} 2 \mathrm{~g}$ of IL used for $0.5 \mathrm{mmol}$ of hydrazone and $1.0 \mathrm{eq}$. maleimide.

To investigate the recyclability of the ionic liquids, we examined the reaction between furfural hydrazone 1a and $N$ ethylmaleimide $\mathbf{2 b}$ further (Table 2).

The yields of $\mathbf{3 b}$ remained high in each of the five experiments, and an excellent recovery of $[\mathrm{bmim}][\mathrm{Cl}]$ was achieved
Table 2 Iterative use of [bmim] [Cl]

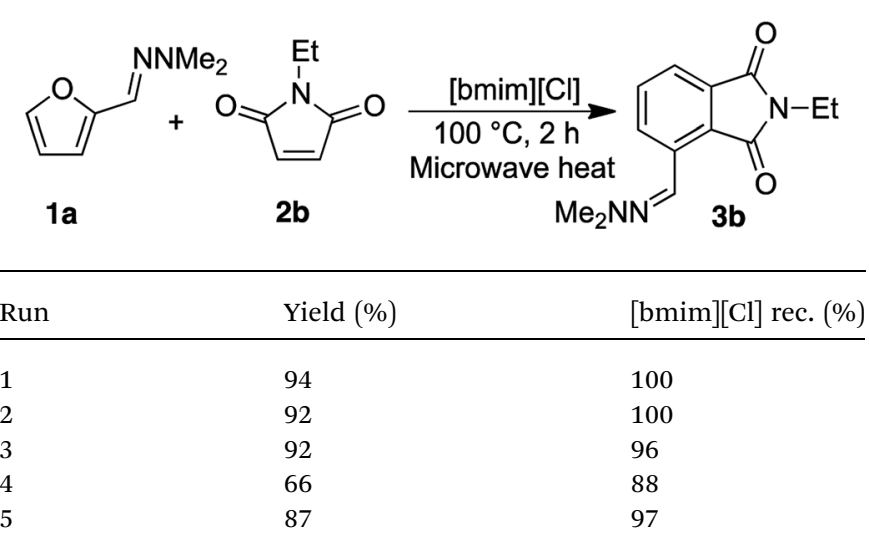

each time. ${ }^{1} \mathrm{H}$ NMR spectroscopic data of the recovered IL indicated that the compound was identical to unused IL. These results clearly highlighted that $[\mathrm{bmim}][\mathrm{Cl}]$ is stable under the reaction conditions used. Thus, the ionic liquids are useful for this conversion, and the efficient recovery and reuse of the ionic liquid is possible under these reaction conditions. Overall, a green conversion of furfurals into phthalimides using ionic liquids has been achieved.

\section{Conclusions}

We have developed a successful protocol employing recyclable ionic liquids for the synthesis of substituted phthalimides from furfurals. The reaction proceeds efficiently under microwave heating with products isolated via a simple work-up procedure requiring a minimal amount of solvent to recover the ionic liquid. The products were recovered by recrystallisation from an acetone/water mixture without the need for chromatographic purification. The recyclability of the ionic liquid was $>95 \%$ with no loss in efficiency over five cycles of use in the reaction. With such straightforward manipulations and the use of ionic liquids as a green solvent, the present protocol will contribute to the development of green chemical syntheses.

\section{Experimental section}

\section{General methods}

All reagents and solvents were purchased and used as supplied unless otherwise stated. All reactions were carried out under the atmosphere of nitrogen unless otherwise stated. All reactions were monitored by thin layer chromatography (TLC) or ${ }^{1} \mathrm{H}$ NMR spectroscopy. TLC plates used were pre-coated with silica gel 60 F254 on aluminium (Merck KGaA) and visualised by UV light $(254 \mathrm{~nm})$ or chemically stained $\left(\mathrm{KMnO}_{4}\right)$. Flash column chromatographic purification was performed using silica gel (Wako Co. Wakosil C-300). ${ }^{1} \mathrm{H}$ NMR and ${ }^{13} \mathrm{C}$ NMR spectra were recorded at $500 \mathrm{MHz}$ (for ${ }^{1} \mathrm{H}$ ) $125 \mathrm{MHz}$ on JEOL lamda-500 or JNMECA 500 Delta2 at ambient temperature, unless otherwise indicated. Deuterated solvents used for NMR spectroscopic 
characterisation were $\mathrm{CDCl}_{3}, \mathrm{CD}_{3} \mathrm{OD}$, or DMSO- $d_{6}$, as stated. Peaks are assigned as singlet $(\mathrm{s})$, doublet $(\mathrm{d})$, triplet $(\mathrm{t})$, quintet (qn), sextet (sx), septet (sept), or multiplet (m). ${ }^{1} \mathrm{H}$ and ${ }^{13} \mathrm{C}$ shifts are reported in parts per million (ppm) and compared against residual solvent signals: $\mathrm{CDCl}_{3}(\delta=7.26 \mathrm{ppm}, \mathrm{s} ; 77.2 \mathrm{ppm}, \mathrm{t})$, $\mathrm{CD}_{3} \mathrm{OD}\left(\delta_{\mathrm{H}} 4.87\right.$, s and 3.31, qn; $\delta_{\mathrm{C}} 49.1 \mathrm{ppm}$, sept $)$, DMSO- $d_{6}\left(\delta_{\mathrm{H}}\right.$ $2.56 \mathrm{ppm}$, qn; $\delta_{\mathrm{C}} 39.5 \mathrm{ppm}$, sept); ${ }^{1} \mathrm{H}$ and ${ }^{13} \mathrm{C}$ NMR shifts relative to TMS were calibrated using residual solvents peak. Infra-red spectra were obtained using a SHIMADZU FTIR-8400S spectrometer, all frequencies were given in reciprocal centimetres $\left(\mathrm{cm}^{-1}\right)$. Melting points were measured on a YANACO MP-J3. High-resolution mass spectra were recorded on a JEOL JMST100LP mass spectrometer. Microwave heating was carried out using a SMW-087 microwave reactor, made by Shikoku Instrument Co. Ltd., $2.45 \mathrm{GHz}$, output $700 \mathrm{~W}$, multimode. The reaction vessel (a $50 \mathrm{~mL}$ glass flask) was placed in the microwave oven (temperature was set at $100{ }^{\circ} \mathrm{C}$ or $120{ }^{\circ} \mathrm{C}$ ). The reaction temperature was monitored by a thermocouple inserted into the reaction mixture and the reaction was stirred under microwave irradiation. Hydrazones $\mathbf{1}$ were prepared as described in the ESI. $\dagger$

4-((2,2-Dimethylhydrazono)methyl)-2-phenylisoindoline-1,3dione 3a. A mixture of hydrazone $1 \mathrm{a}(96 \mathrm{mg}, 0.70 \mathrm{mmol})$ and $\mathrm{N}$ phenylmaleimide (182 mg, $1.05 \mathrm{mmol})$ in [bmim] [Cl] (1.0324 g) was heated at $100{ }^{\circ} \mathrm{C}$ for $2 \mathrm{~h}$ in a microwave. Water $(20 \mathrm{~mL})$ was added and the reaction mixture was extracted with EtOAc $(3 \times$ $20 \mathrm{~mL}$ ). The combined organic extracts were washed with water, brine, dried $\left(\mathrm{Na}_{2} \mathrm{SO}_{4}\right)$, filtered, and concentrated in vacuo. The residue was recrystallised from acetone and cold water to obtain the product as a yellow solid (170 $\mathrm{mg}, 83 \%)$. The remaining water phase was concentrated and dried to recover the ionic liquid (1.0237 $\mathrm{g}, 99 \%$ recovery).

Yellow solid; mp 221-222 ${ }^{\circ} \mathrm{C} ;{ }^{1} \mathrm{H}$ NMR (500 $\mathrm{MHz}, \mathrm{CDCl}_{3}$ ) $\delta 8.31(\mathrm{~d}, J=8.1 \mathrm{~Hz}, 1 \mathrm{H}, \mathrm{NNCHCCH}), 8.15(\mathrm{~s}, 1 \mathrm{H}, \mathrm{NNCH}), 7.72$ (d, $J=7.2 \mathrm{~Hz}, 1 \mathrm{H}, \mathrm{NCHCCHCHCH}), 7.64(\mathrm{t}, J=7.8 \mathrm{~Hz}, 1 \mathrm{H}$, $\mathrm{NCHCCHCH}), 7.53-7.46$ (m, 2H, Ph), 7.44-7.37 (m, 3H, Ph), 3.13 $\left(\mathrm{s}, 6 \mathrm{H}, 2 \times \mathrm{CH}_{3}\right) ;{ }^{13} \mathrm{C} \mathrm{NMR}\left(125 \mathrm{MHz}, \mathrm{CDCl}_{3}\right) \delta 168.5,167.5$, $137.0,133.8,132.1,131.8,129.4$, 129.2, 128.1, 126.8, 124.3, 124.1, 121.3, 42.7; HMRS: found (ESI): $[\mathrm{M}+\mathrm{H}]^{+} 294.12390$ $\mathrm{C}_{17} \mathrm{H}_{16} \mathrm{~N}_{3} \mathrm{O}_{2}$, requires 294.12425; data in agreement with the literature. ${ }^{4}$

4-((2,2-Dimethylhydrazono)methyl)-2-ethylisoindoline-1,3-

dione 3b. A mixture of hydrazone $1 \mathrm{a}(96 \mathrm{mg}, 0.70 \mathrm{mmol})$ and $N$ ethylmaleimide $(131 \mathrm{mg}, 1.05 \mathrm{mmol})$ in [bmim] [Cl] (1.0291 g) was heated at $100^{\circ} \mathrm{C}$ by microwave irradiation for $2 \mathrm{~h}$. Water $(20$ $\mathrm{mL}$ ) was added and the reaction mixture was extracted with EtOAc $(3 \times 15 \mathrm{~mL})$. The combined organic extracts were washed with water, brine, dried $\left(\mathrm{Na}_{2} \mathrm{SO}_{4}\right)$, filtered, and concentrated in vacuo. The residue was further recrystallised from acetone and cold water and the product collected by suction-filtration as a yellow solid (161 $\mathrm{mg}, 94 \%)$. The remaining water phase was concentrated and dried to recover the ionic liquid $(1.0254 \mathrm{~g}$, 100\% recovery).

Yellow solid; mp $141-143{ }^{\circ} \mathrm{C}$ [lit. $\left.{ }^{4} 142-143{ }^{\circ} \mathrm{C}\right] ;{ }^{1} \mathrm{H}$ NMR $(500$ $\left.\mathrm{MHz} \mathrm{CDCl}_{3}\right) \delta 8.22(\mathrm{~d}, J=7.7 \mathrm{~Hz}, 1 \mathrm{H}, \mathrm{N}=\mathrm{CHCCH}), 8.11(\mathrm{~s}, 1 \mathrm{H}$, $\mathrm{N}=\mathrm{CH}), 7.61(\mathrm{~d}, J=7.7 \mathrm{~Hz}, 1 \mathrm{H}, \mathrm{N}=\mathrm{CHCCHCHCH}), 7.55(\mathrm{t}, J=$ $7.7 \mathrm{~Hz}, 1 \mathrm{H}, \mathrm{N}=\mathrm{CHCCHCH}), 3.72\left(\mathrm{q}, J=7.3 \mathrm{~Hz}, 2 \mathrm{H}, \mathrm{CH}_{3} \mathrm{CH}_{2}\right.$ ), $3.12\left(\mathrm{~s}, 6 \mathrm{H}, 2 \times \mathrm{CH}_{3}\right), 1.26\left(\mathrm{t}, J=7.3 \mathrm{~Hz}, 3 \mathrm{H}, \mathrm{CH}_{3} \mathrm{CH}_{2}\right) ;{ }^{13} \mathrm{C} \mathrm{NMR}$ $\left(125 \mathrm{MHz}, \mathrm{CDCl}_{3}\right) \delta 169.5,168.5,136.2,133.4,132.6,129.0$, 124.9, 124.7, 120.9, 42.7, 32.9, 14.1; HMRS: found (ESI): $[\mathrm{M}+\mathrm{H}]^{+}$ 268.1068 $\mathrm{C}_{13} \mathrm{H}_{15} \mathrm{~N}_{3} \mathrm{NaO}_{2}$, requires 268.1062; data in agreement with the literature. ${ }^{4}$

4-((2,2-Dimethylhydrazino)methyl)-2-propylisoindoline-1,3dione 3c. A mixture of hydrazone $1 \mathrm{a}(55 \mathrm{mg}, 0.40 \mathrm{mmol})$ and $\mathrm{N}$ propylmaleimide (112 $\mathrm{mg}, 0.81 \mathrm{mmol})$ in [bmim] [Cl] (1.8394 g) was heated at $100{ }^{\circ} \mathrm{C}$ by microwave irradiation for $2 \mathrm{~h}$. Water $(10$ $\mathrm{mL}$ ) was added to the reaction mixture and the resulting mixture was extracted with EtOAc $(5 \times 10 \mathrm{~mL})$. The combined organic extracts were washed with water, brine, dried $\left(\mathrm{Na}_{2} \mathrm{SO}_{4}\right)$, filtered, and concentrated in vacuo. The residue was recrystallised from acetone and cold water and the product collected by suction-filtration as a yellow solid ( $75 \mathrm{mg}, 73 \%)$. The remaining water phase was concentrated and dried to recover the ionic liquid (1.7979 $\mathrm{g}, 98 \%$ recovery).

Yellow solid; mp $121-122^{\circ} \mathrm{C}$; $\nu_{\max }$ (solid per cm) 2964, 1703, 1552; ${ }^{1} \mathrm{H}$ NMR $\left(500 \mathrm{MHz}, \mathrm{CDCl}_{3}\right) \delta 8.20(\mathrm{~d}, J=8.0 \mathrm{~Hz}, 1 \mathrm{H}$, $\mathrm{NNCHCCH}), 8.10(\mathrm{~s}, 1 \mathrm{H}, \mathrm{NNCH}), 7.59(\mathrm{~d}, J=7.2 \mathrm{~Hz}, 1 \mathrm{H}$, $\mathrm{NNCCHCHCH}), 7.54(\mathrm{t}, J=7.6 \mathrm{~Hz}, 1 \mathrm{H}, \mathrm{NNCHCCHCH}), 3.61(\mathrm{t}, J$ $\left.=7.3 \mathrm{~Hz}, 2 \mathrm{H}, \mathrm{NCH}_{2} \mathrm{CH}_{2} \mathrm{CH}_{3}\right), 3.11\left(\mathrm{~s}, 6 \mathrm{H}, 2 \times \mathrm{CH}_{3}\right), 1.72-1.65$ ( $\left.\mathrm{m}, 2 \mathrm{H}, \mathrm{NCH}_{2} \mathrm{CH}_{2} \mathrm{CH}_{3}\right), 0.94$ (t, $J=7.3 \mathrm{~Hz}, 3 \mathrm{H}, \mathrm{NCH}_{2} \mathrm{CH}_{2} \mathrm{CH}_{3}$ ); ${ }^{13} \mathrm{C}$ NMR $\left(125 \mathrm{MHz}, \mathrm{CDCl}_{3}\right) \delta 169.7,168.7,136.3$, 133.3, 132.4, 128.9, 124.7, 124.6, 120.8, 42.7, 39.5, 22.1, 11.5; HRMS: found (ESI): $[\mathrm{M}+\mathrm{H}]^{+} 260.13883 \mathrm{C}_{14} \mathrm{H}_{18} \mathrm{~N}_{3} \mathrm{O}_{2}$, requires 260.13990.

\section{4-((2,2-Dimethylhydrazino)methyl)-2-butylisoindoline-1,3-}

dione 3d. A mixture of hydrazone $1 \mathrm{a}(96 \mathrm{mg}, 0.69 \mathrm{mmol})$ and $N$ butylmaleimide (103 mg, $0.67 \mathrm{mmol})$ in [bmim][Cl] (2.01 g) was heated at $100{ }^{\circ} \mathrm{C}$ by microwave irradiation for $2 \mathrm{~h}$. Water $(10 \mathrm{~mL})$ was added to the reaction mixture and the resulting mixture was extracted with EtOAc $(5 \times 10 \mathrm{~mL})$. The combined organic extracts were washed with water, brine, dried $\left(\mathrm{Na}_{2} \mathrm{SO}_{4}\right)$, filtered, and concentrated in vacuo. The residue was recrystallised from acetone and cold water and the product collected by suctionfiltration as a yellow solid ( $88 \mathrm{mg}, 48 \%$ ). The remaining water phase was concentrated and dried to recover the ionic liquid (1.85 $\mathrm{g}, 92 \%$ recovery).

Yellow solid; mp 91-92 ${ }^{\circ} \mathrm{C} ;{ }^{1} \mathrm{H}$ NMR $\left(500 \mathrm{MHz}, \mathrm{CDCl}_{3}\right) \delta 8.22$ (d, $J=8.0 \mathrm{~Hz}, 1 \mathrm{H}, \mathrm{NNCHCCH}), 8.12(\mathrm{~s}, 1 \mathrm{H}, \mathrm{NNCH}), 7.61(\mathrm{~d}, J=$ $7.2 \mathrm{~Hz}, 1 \mathrm{H}, \mathrm{NNCCHCHCH}), 7.59-7.50$ (m, 1H, NNCCHCH), 3.66 (t, $\left.J=7.3 \mathrm{~Hz}, 2 \mathrm{H}, \mathrm{NCH}_{2} \mathrm{CH}_{2} \mathrm{CH}_{2} \mathrm{CH}_{3}\right), 3.12\left(\mathrm{~s}, 6 \mathrm{H}, 2 \times \mathrm{CH}_{3}\right)$, 1.68-1.62 (m, 2H, $\left.\mathrm{NCH}_{2} \mathrm{CH}_{2} \mathrm{CH}_{2} \mathrm{CH}_{3}\right), 1.38-1.32(\mathrm{~m}, 2 \mathrm{H}$, $\mathrm{NCH}_{2} \mathrm{CH}_{2} \mathrm{CH}_{2} \mathrm{CH}_{3}$ ), $0.94\left(\mathrm{t}, J=7.4 \mathrm{~Hz}, 3 \mathrm{H}, \mathrm{NCH}_{2} \mathrm{CH}_{2} \mathrm{CH}_{2} \mathrm{CH}_{3}\right.$ ); ${ }^{13} \mathrm{C}$ NMR (125 MHz, $\left.\mathrm{CDCl}_{3}\right) \delta 169.6,168.6,136.3,133.3,132.4$, 128.9, 124.8, 124.6, 120.8, 42.7, 37.7, 31.1, 20.2, 13.8; HRMS: found (ESI): $[\mathrm{M}+\mathrm{H}]^{+} 274.15652 \mathrm{C}_{15} \mathrm{H}_{20} \mathrm{~N}_{3} \mathrm{O}_{2}$, requires 274.15555.

4-((2,2-Dimethylhydrazino)methyl)-2-pentylisoindoline-1,3dione 3e. A mixture of hydrazone $1 \mathrm{a}(73 \mathrm{mg}, 0.53 \mathrm{mmol})$ and $N$ pentylmaleimide (102 $\mathrm{mg}, 0.61 \mathrm{mmol})$ in [bmim] [Cl] (1.99 g) was heated at $100{ }^{\circ} \mathrm{C}$ by microwave irradiation for $2 \mathrm{~h}$. Water $(10$ $\mathrm{mL}$ ) was added to the reaction mixture and the resulting mixture was extracted with EtOAc $(5 \times 10 \mathrm{~mL})$. The combined organic extracts were washed with water, brine, dried $\left(\mathrm{Na}_{2} \mathrm{SO}_{4}\right)$, filtered, and concentrated in vacuo. The residue was recrystallised from acetone and cold water and the product collected by 
suction-filtration as a yellow solid (79 $\mathrm{mg}, 52 \%)$. The remaining water phase was concentrated and dried to recover the ionic liquid (1.95 g, 92\% recovery).

Yellow solid; mp $74-75{ }^{\circ} \mathrm{C} ; \nu_{\max }$ (solid per cm) 2995, 1697, 1548; ${ }^{1} \mathrm{H}$ NMR (500 MHz, $\left.\mathrm{CDCl}_{3}\right) \delta 8.20(\mathrm{~d}, J=8.0 \mathrm{~Hz}, 1 \mathrm{H}$, $\mathrm{NNCHCCH}), \quad 8.09(\mathrm{~s}, 1 \mathrm{H}, \mathrm{NNCH}), 7.61-7.56(\mathrm{~m}, 1 \mathrm{H}$, $\mathrm{NNCCHCHCH}), 7.53(\mathrm{t}, J=7.7 \mathrm{~Hz}, 1 \mathrm{H}, \mathrm{NNCCHCH}), 3.66-3.58$ $\left(\mathrm{m}, 2 \mathrm{H}, \mathrm{NCH}_{2} \mathrm{CH}_{2} \mathrm{CH}_{2} \mathrm{CH}_{2} \mathrm{CH}_{3}\right), 3.10\left(\mathrm{~s}, 6 \mathrm{H}, 2 \times \mathrm{CH}_{3}\right), 1.68-1.60$ (m, 2H, $\mathrm{NCH}_{2} \mathrm{CH}_{2} \mathrm{CH}_{2} \mathrm{CH}_{2} \mathrm{CH}_{3}$ ), 1.37-1.29 (m, 4H, $\mathrm{NCH}_{2} \mathrm{CH}_{2}-$ $\mathrm{CH}_{2} \mathrm{CH}_{2} \mathrm{CH}_{3}$ ), 0.87 (t, $\left.J=7.1 \mathrm{~Hz}, 3 \mathrm{H}, \mathrm{NCH}_{2} \mathrm{CH}_{2} \mathrm{CH}_{2} \mathrm{CH}_{2} \mathrm{CH}_{3}\right) ;{ }^{13} \mathrm{C}$ NMR (125 MHz, $\mathrm{CDCl}_{3}$ ) $\delta 169.6,168.6,136.3,133.3,132.4,128.9$, 124.8, 124.6, 120.8, 42.7, 38.0, 29.1, 28.4, 22.4, 14.1; HRMS: found (ESI): $[\mathrm{M}+\mathrm{H}]^{+} 288.17265 \mathrm{C}_{16} \mathrm{H}_{22} \mathrm{~N}_{3} \mathrm{O}_{2}$, requires 288.17120.

4-((2,2-Dimethylhydrazino)methyl)-2-hexylisoindoline-1,3-

dione 3f. A mixture of hydrazone $1 \mathrm{a}(67.3 \mathrm{mg}, 0.488 \mathrm{mmol})$ and $N$-hexylmaleimide $(129.0 \mathrm{mg}, 0.705 \mathrm{mmol})$ in [bmim] [Cl] $(1.083$ g) was heated at $100{ }^{\circ} \mathrm{C}$ by microwave irradiation for $2 \mathrm{~h}$. Water $(10 \mathrm{~mL})$ was added to the reaction mixture and the resulting mixture was extracted with EtOAc $(5 \times 10 \mathrm{~mL})$. The combined organic extracts were washed with water, brine, dried $\left(\mathrm{Na}_{2} \mathrm{SO}_{4}\right)$, filtered, and concentrated in vacuo. The residue was recrystallised from acetone and cold water and the product collected by suction-filtration as a yellow solid $(88.7 \mathrm{mg}, 60 \%)$. The remaining water phase was concentrated and dried to recover the ionic liquid $(1.029 \mathrm{~g}, 95 \%$ recovery).

Yellow solid; mp $73-74{ }^{\circ} \mathrm{C} ; \nu_{\max }$ (solid per cm) 2929, 1699, 1550; ${ }^{1} \mathrm{H}$ NMR $\left(500 \mathrm{MHz}, \mathrm{CDCl}_{3}\right) \delta 8.18(\mathrm{~d}, J=7.8 \mathrm{~Hz}, 1 \mathrm{H}$, $\mathrm{NNCHCCH}), 8.08(\mathrm{~s}, 1 \mathrm{H}, \mathrm{NN}=\mathrm{CH}), 7.57(\mathrm{~d}, J=8.2 \mathrm{~Hz}, 1 \mathrm{H}$, $\mathrm{NNCCHCHCH}), 7.52(\mathrm{t}, J=7.6 \mathrm{~Hz}, 1 \mathrm{H}, \mathrm{NNCCHCH}), 3.62(\mathrm{~d}, J=$ $\left.7.4 \mathrm{~Hz}, 2 \mathrm{H}, \mathrm{NCH}_{2} \mathrm{CH}_{2} \mathrm{CH}_{2} \mathrm{CH}_{2} \mathrm{CH}_{2} \mathrm{CH}_{3}\right), 3.09\left(\mathrm{~s}, 6 \mathrm{H}, 2 \times \mathrm{CH}_{3}\right)$, 1.63 (p, $J=7.4 \mathrm{~Hz}, 2 \mathrm{H}, \mathrm{NCH}_{2} \mathrm{CH}_{2} \mathrm{CH}_{2} \mathrm{CH}_{2} \mathrm{CH}_{2} \mathrm{CH}_{3}$ ), 1.36-1.13 $\left(\mathrm{m}, 6 \mathrm{H}, \mathrm{NCH}_{2} \mathrm{CH}_{2} \mathrm{CH}_{2} \mathrm{CH}_{2} \mathrm{CH}_{2} \mathrm{CH}_{3}\right), 0.85$ (t, $J=6.9 \mathrm{~Hz}, 3 \mathrm{H}, \mathrm{N}-$ $\left.\left(\mathrm{CH}_{2}\right)_{5}-\mathrm{CH}_{3}\right) ;{ }^{13} \mathrm{C} \mathrm{NMR}\left(125 \mathrm{MHz}, \mathrm{CDCl}_{3}\right) \delta$ 169.6, 168.6, 136.3, 133.3, 132.4, 128.9, 124.8, 124.6, 120.8, 42.7, 38.0, 31.5, 28.7, 26.7, 22.6, 14.1; HRMS: found (ESI): $[\mathrm{M}+\mathrm{Na}]^{+} 324.16711$ $\mathrm{C}_{17} \mathrm{H}_{23} \mathrm{~N}_{3} \mathrm{O}_{2} \mathrm{Na}$, requires 324.16880.

4-((2,2-Dimethylhydrazino)methyl)-2-heptylisoindoline-1,3dione $3 \mathrm{~g}$. A mixture of hydrazone $1 \mathrm{a}(76 \mathrm{mg}, 0.55 \mathrm{mmol})$ and $\mathrm{N}$ heptylmaleimide $(105 \mathrm{mg}, 0.54 \mathrm{mmol})$ in [bmim] [Cl] $(1.98 \mathrm{~g})$ was heated at $100{ }^{\circ} \mathrm{C}$ by microwave irradiation for $2 \mathrm{~h}$. Water $(10$ $\mathrm{mL}$ ) was added to the reaction mixture and the resulting mixture was extracted with EtOAc $(5 \times 10 \mathrm{~mL})$. The combined organic extracts were washed with water, brine, dried $\left(\mathrm{Na}_{2} \mathrm{SO}_{4}\right)$, filtered, and concentrated in vacuo. The residue was recrystallised from acetone and cold water and the product collected by suction-filtration as a yellow solid ( $99 \mathrm{mg}, 58 \%$ ). The remaining water phase was concentrated and dried to recover the ionic liquid (1.94 g, 98\% recovery).

Yellow solid; mp 55-56 ${ }^{\circ} \mathrm{C} ; \nu_{\max }$ (solid per cm) 2928, 1699, 1550; ${ }^{1} \mathrm{H}$ NMR $\left(500 \mathrm{MHz}, \mathrm{CDCl}_{3}\right) \delta 8.21(\mathrm{~d}, J=8.0 \mathrm{~Hz}, 1 \mathrm{H}$, $\mathrm{NNCHCCH}), 8.12(\mathrm{~s}, 1 \mathrm{H}, \mathrm{NNCH}), 7.60(\mathrm{~d}, J=7.3 \mathrm{~Hz}, 1 \mathrm{H}$, $\mathrm{NNCHCCHCHCH}), 7.56$ (d, $J=7.6 \mathrm{~Hz}, 1 \mathrm{H}, \mathrm{NNCHCCHCH})$, 3.68-3.60 (m, 2H, $\left.\mathrm{NCH}_{2}\left(\mathrm{CH}_{2}\right)_{5} \mathrm{CH}_{3}\right), 3.11\left(\mathrm{~s}, 6 \mathrm{H}, 2 \times \mathrm{CH}_{3}\right), 1.70-$ $1.61\left(\mathrm{~m}, 2 \mathrm{H}, \mathrm{NCH}_{2} \mathrm{CH}_{2}\left(\mathrm{CH}_{2}\right)_{4} \mathrm{CH}_{3}\right), 1.36-1.29\left(\mathrm{~m}, 4 \mathrm{H}, 2 \times \mathrm{CH}_{2}\right)$, 1.29-1.18 (m, 4H, $\left.2 \times \mathrm{CH}_{2}\right), 0.86\left(\mathrm{t}, J=6.8 \mathrm{~Hz}, 3 \mathrm{H}, \mathrm{N}\left(\mathrm{CH}_{2}\right)_{6} \mathrm{CH}_{3}\right)$; ${ }^{13} \mathrm{C}$ NMR $\left(125 \mathrm{MHz}, \mathrm{CDCl}_{3}\right) \delta 169.4,168.4,136.2,133.1,132.4$, 128.7, 124.6, 124.4, 120.6, 42.5, 37.9, 31.8, 28.9, 28.7, 26.9, 22.6,
14.1.; HRMS: found (ESI): $[\mathrm{M}+\mathrm{H}]^{+} 316.20250 \mathrm{C}_{18} \mathrm{H}_{26} \mathrm{~N}_{3} \mathrm{O}_{2}$, requires 316.20250 .

4-((2,2-Dimethylhydrazino)methyl)-2-octylisoindoline-1,3-

dione $3 \mathrm{~h}$. A mixture of hydrazone $1 \mathrm{a}(99.7 \mathrm{mg}, 0.72 \mathrm{mmol})$ and $N$-octylmaleimide $(210 \mathrm{mg}, 1.00 \mathrm{mmol})$ in [bmim] [Cl] $(1.012 \mathrm{~g})$ was heated at $100{ }^{\circ} \mathrm{C}$ by microwave irradiation for $2 \mathrm{~h}$. Water $(10$ $\mathrm{mL}$ ) was added to the reaction mixture and the resulting mixture was extracted with EtOAc $(5 \times 10 \mathrm{~mL})$. The combined organic extracts were washed with water, brine, dried $\left(\mathrm{Na}_{2} \mathrm{SO}_{4}\right)$, filtered, and concentrated in vacuo. The residue was recrystallised from acetone and cold water and the product collected by suction-filtration as a yellow solid (119.3 $\mathrm{mg}$, 50\%). The remaining water phase was concentrated and dried to recover the ionic liquid $(0.958 \mathrm{~g}, 95 \%$ recovery).

Yellow solid; mp $50-51{ }^{\circ} \mathrm{C} ; \nu_{\max }$ (solid per $\mathrm{cm}$ ) 2926, 1703, 1550; ${ }^{1} \mathrm{H}$ NMR $\left(500 \mathrm{MHz}, \mathrm{CDCl}_{3}\right) \delta 8.21(\mathrm{~d}, J=7.9 \mathrm{~Hz}, 1 \mathrm{H}), 8.11$ $(\mathrm{s}, 1 \mathrm{H}), 7.60(\mathrm{~d}, J=7.1 \mathrm{~Hz}, 1 \mathrm{H}), 7.54(\mathrm{t}, J=7.6 \mathrm{~Hz}, 1 \mathrm{H}), 3.63(\mathrm{t}, J$ $=7.3 \mathrm{~Hz}, 2 \mathrm{H}), 3.11(\mathrm{~s}, 6 \mathrm{H}), 1.65(\mathrm{~m}, J=7.3 \mathrm{~Hz}, 2 \mathrm{H}), 1.34-1.22$ $(\mathrm{m}, 10 \mathrm{H}), 0.85(\mathrm{t}, J=6.7 \mathrm{~Hz}, 3 \mathrm{H}) ;{ }^{13} \mathrm{C} \mathrm{NMR}\left(125 \mathrm{MHz}, \mathrm{CDCl}_{3}\right)$ $\delta$ 169.6, 168.6, 136.3, 133.3, 132.5, 128.9, 124.8, 124.7, 120.8, 42.7, 38.0, 31.9, 29.3, 29.3, 28.8, 27.0, 22.7, 14.2; HRMS: found (ESI): $[\mathrm{M}+\mathrm{Na}]^{+} 352.19881 \mathrm{C}_{19} \mathrm{H}_{27} \mathrm{~N}_{3} \mathrm{O}_{2} \mathrm{Na}$, requires 352.20010.

4-((2,2-Dimethylhydrazino)methyl)-2-nonylisoindoline-1,3-

dione $3 i$. A mixture of hydrazone $1 \mathrm{a}(73 \mathrm{mg}, 0.53 \mathrm{mmol})$ and $N$ nonylmaleimide (118 mg, $0.53 \mathrm{mmol})$ in [bmim] [Cl] (2.00 g) was heated at $100{ }^{\circ} \mathrm{C}$ by microwave irradiation for $2 \mathrm{~h}$. Water $(10 \mathrm{~mL})$ was added to the reaction mixture and the resulting mixture was extracted with EtOAc $(5 \times 10 \mathrm{~mL})$. The combined organic extracts were washed with water, brine, dried $\left(\mathrm{Na}_{2} \mathrm{SO}_{4}\right)$, filtered, and concentrated in vacuo. The residue was recrystallised from acetone and cold water and the product collected by suctionfiltration as a yellow solid (62 $\mathrm{mg}, 34 \%$ ). The remaining water phase was concentrated and dried to recover the ionic liquid (1.98 $\mathrm{g}, 99 \%$ recovery).

Yellow solid; mp $42-43{ }^{\circ} \mathrm{C} ; \nu_{\max }$ (solid per cm) 2924, 1701, 1550; ${ }^{1} \mathrm{H} \mathrm{NMR}\left(500 \mathrm{MHz}, \mathrm{CDCl}_{3}\right) \delta 8.21$ (d, $J=8.0 \mathrm{~Hz}, 1 \mathrm{H}$, $\mathrm{NNCHCCH}), 8.11(\mathrm{~s}, 1 \mathrm{H}, \mathrm{NNCH}), 7.60(\mathrm{~d}, J=7.2 \mathrm{~Hz}, 1 \mathrm{H}$, $\mathrm{NNCHCCHCHCH}), 7.55$ (t, $J=7.6 \mathrm{~Hz}, 1 \mathrm{H}, \mathrm{NNCHCCHCH}), 3.64$ $\left(\mathrm{t}, J=7.3 \mathrm{~Hz}, 2 \mathrm{H}, \mathrm{NCH}_{2}\left(\mathrm{CH}_{2}\right)_{8} \mathrm{CH}_{3}\right), 3.11\left(\mathrm{~s}, 6 \mathrm{H}, 2 \times \mathrm{CH}_{3}\right), 1.66-$ $1.62\left(\mathrm{~m}, 2 \mathrm{H}, \mathrm{NCH}_{2} \mathrm{CH}_{2}\left(\mathrm{CH}_{2}\right)_{6} \mathrm{CH}_{3}\right), 1.37-1.22\left(\mathrm{~m}, 4 \mathrm{H}, 2 \times \mathrm{CH}_{2}\right)$, 1.27-1.22 (m, 8H, $\left.4 \times \mathrm{CH}_{2}\right), 0.86\left(\mathrm{t}, J=6.9 \mathrm{~Hz}, 3 \mathrm{H}, \mathrm{NCH}_{2}(-\right.$ $\left.\left.\mathrm{CH}_{2}\right)_{8} \mathrm{CH}_{3}\right) ;{ }^{13} \mathrm{C}$ NMR $\left(125 \mathrm{MHz}, \mathrm{CDCl}_{3}\right) \delta$ 169.6, 168.6, 136.3, 134.1, 133.3, 132.4, 128.9, 124.6, 120.8, 42.7, 38.0, 31.9, 29.5, 29.3, 29.2, 28.8, 27.0, 22.8, 14.2; HRMS: found (ESI): $[\mathrm{M}+\mathrm{Na}]^{+}$ 366.21742 $\mathrm{C}_{20} \mathrm{H}_{29} \mathrm{~N}_{3} \mathrm{O}_{2} \mathrm{Na}$, requires 366.21575.

4-((2,2-Dimethylhydrazono)methyl)isoindoline-1,3-dione $3 \mathbf{j}$. A mixture of hydrazone $3 \mathbf{a}(96 \mathrm{mg}, 0.70 \mathrm{mmol})$ and maleimide (103 mg, $1.05 \mathrm{mmol})$ in [bmim][Cl] (1.0085 g) was heated at $100{ }^{\circ} \mathrm{C}$ by microwave irradiation for $2 \mathrm{~h}$. Water $(20 \mathrm{~mL})$ was added and the reaction mixture was extracted with EtOAc $(4 \times$ $20 \mathrm{~mL}$ ). The combined organic extracts were washed with water, brine, dried $\left(\mathrm{Na}_{2} \mathrm{SO}_{4}\right)$, filtered, and concentrated in vacuo. The residue was recrystallised from acetone and cold water and the product collected by suction-filtration as a yellow solid $(81 \mathrm{mg}$, $53 \%$ ). The remaining water phase was concentrated and dried to recover the ionic liquid ( $0.9180 \mathrm{~g}, 91 \%$ recovery). 
Yellow solid; mp 240-241 ${ }^{\circ} \mathrm{C} ;{ }^{1} \mathrm{H}$ NMR (500 $\mathrm{MHz}, \mathrm{CDCl}_{3}$ ) $\delta 8.27$ (d, $J=7.2 \mathrm{~Hz}, 1 \mathrm{H}, \mathrm{NNCHCCH}), 8.06$ (s, 1H, NCH), 7.63$7.58(\mathrm{~m}, 3 \mathrm{H}, \mathrm{NNCHCCHCH}, \mathrm{NNCHCCHCHCH}$ and $\mathrm{N}-\mathrm{H}), 3.13$ $\left(\mathrm{s}, 6 \mathrm{H}, 2 \times \mathrm{CH}_{3}\right) ;{ }^{13} \mathrm{C} \mathrm{NMR}\left(125 \mathrm{MHz}, \mathrm{CDCl}_{3}\right) \delta 169.0,168.0$, 137.0, 133.8, 133.0, 129.3, 125.1, 124.3, 121.2, 42.8; HRMS found (ESI): $[\mathrm{M}+\mathrm{Na}]^{+} 240.0743 \mathrm{C}_{11} \mathrm{H}_{11} \mathrm{~N}_{3} \mathrm{NaO}_{2}$, requires 240.0749 .

2-Cyclohexyl-4-((2,2-dimethylhydrazono)methyl)isoindoline1,3-dione 3k. A mixture of hydrazone 1a (45 mg, $0.33 \mathrm{mmol}, 1.0$ eq.) and $N$-cyclohexylmaleimide ( $88 \mathrm{mg}, 0.49 \mathrm{mmol}, 1.5 \mathrm{eq}$.$) in$ [bmim $][\mathrm{Cl}](0.517 \mathrm{~g})$ was heated at $100{ }^{\circ} \mathrm{C}$ by microwave irradiation for $2 \mathrm{~h}$. Water $(10 \mathrm{~mL})$ was added to the reaction mixture and the resulting mixture was extracted with EtOAc $(3 \times 10 \mathrm{~mL})$. The combined organic phase was washed with brine, dried over $\mathrm{Na}_{2} \mathrm{SO}_{4}$ and filtered. The filtrate was concentrated to give a crude product which was recrystallised from water-acetone to give $3 \mathbf{k}$ as a yellow solid (67 $\mathrm{mg}, 68 \%$ ). The remaining water phase was concentrated and dried to recover the ionic liquid (0.509 $\mathrm{g}, 98 \%$ recovery).

Yellow solid; mp 187-189 ${ }^{\circ} \mathrm{C}$; $\nu_{\max }$ (solid per $\mathrm{cm}$ ) 2951, 2918, 2851, 1755, 1697, 1549, 1464; ${ }^{1} \mathrm{H}$ NMR (500 MHz, $\left.\mathrm{CDCl}_{3}\right) \delta 8.20$ (d, $J=7.5 \mathrm{~Hz}, 1 \mathrm{H}, \mathrm{NNCHCCH}), 8.12(\mathrm{~s}, 1 \mathrm{H}, \mathrm{NCH}), 7.58(\mathrm{~d}, J=$ $7.5 \mathrm{~Hz}, 1 \mathrm{H}, \mathrm{NNCCHCHCH}), 7.54$ (t, $J=7.5 \mathrm{~Hz}, 1 \mathrm{H}, \mathrm{NNCCHCH})$, 4.13-4.03 (m, 1H, CONCH), 3.11 (s, 6H, $\left.2 \times \mathrm{CH}_{3}\right), 2.25-2.13(\mathrm{~m}$, $\left.2 \mathrm{H}, \mathrm{CyCH}_{2}\right), 1.87-1.84\left(\mathrm{~m}, 2 \mathrm{H}, \mathrm{CyCH}_{2}\right), 1.73-1.71(\mathrm{~m}, 2 \mathrm{H}$, $\mathrm{CyCH}_{2}$ ), 1.67 (s, 1H, $\mathrm{CyCH}_{2}$ ), 1.43-1.17 (m, 2H, $\left.\mathrm{CyCH}_{2}\right), 0.98-$ $0.74\left(\mathrm{~m}, 1 \mathrm{H}, \mathrm{CyCH}_{2}\right) ;{ }^{13} \mathrm{C} \mathrm{NMR}\left(125 \mathrm{MHz}, \mathrm{CDCl}_{3}\right) \delta 169.8,168.6$, 136.1, 133.2, 132.3, 128.9, 124.7, 120.7, 50.8, 42.7, 30.0, 29.8, 26.2, 25.3; HRMS: found (ESI): $[\mathrm{M}+\mathrm{H}]^{+} 300.1727 \mathrm{C}_{17} \mathrm{H}_{22} \mathrm{~N}_{3} \mathrm{O}_{2}$, requires 300.1707 .

2-Allyl-4-((2,2-dimethylhydrazono)methyl)isoindoline-1,3-

dione 31. A mixture of hydrazone $1 \mathrm{a}(101 \mathrm{mg}, 0.73 \mathrm{mmol})$ and $N$ allylmaleimide (147 mg, $1.05 \mathrm{mmol})$ in [bmim][Cl] (1.0070 g) was heated at $100{ }^{\circ} \mathrm{C}$ by microwave irradiation for $2 \mathrm{~h}$. Water $(20 \mathrm{~mL})$ was added and the reaction mixture was extracted with EtOAc (5 $\times 10 \mathrm{~mL}$ ). The combined organic extracts were washed with water, brine, dried $\left(\mathrm{Na}_{2} \mathrm{SO}_{4}\right)$, filtered, and concentrated in vacuo. The residue was recrystallised from acetone and cold water and the product collected by suction-filtration as a yellow solid (175 mg, 93\%). The remaining water phase was concentrated and dried to recover the ionic liquid ( $0.9768 \mathrm{~g}, 97 \%$ recovery).

Yellow solid; mp $128-129{ }^{\circ} \mathrm{C}$; $\nu_{\max }$ (solid per cm) 2986, 2924, 2889, 2359, 2332, 1759, 1701, 1547, 1427; ${ }^{1} \mathrm{H}$ NMR $(500 \mathrm{MHz}$, $\left.\mathrm{CDCl}_{3}\right) \delta 8.22(\mathrm{~d}, J=8.0 \mathrm{~Hz}, 1 \mathrm{H}, \mathrm{NNCHCCH}), 8.08(\mathrm{~s}, 1 \mathrm{H}$, $\mathrm{NNCH}), 7.61$ (d, $J=7.2 \mathrm{~Hz}, 1 \mathrm{H}, \mathrm{NNCHCCHCHCH}), 7.55(\mathrm{t}, J=$ $7.7 \mathrm{~Hz}, 1 \mathrm{H}, \mathrm{NNCHCCHCH}), 5.94-5.82(\mathrm{~m}, 1 \mathrm{H}, \mathrm{CHH}=\mathrm{CH}), 5.30-$ $5.21(\mathrm{~m}, 1 \mathrm{H}, \mathrm{CHH}=\mathrm{CH}), 5.18\left(\mathrm{~d}, J=10.2 \mathrm{~Hz}, 1 \mathrm{H}, \mathrm{CH}_{2}=\mathrm{CH}\right)$, $4.26\left(\mathrm{~d}, J=5.5 \mathrm{~Hz}, 2 \mathrm{H}, \mathrm{NCH}_{2}\right), 3.11\left(\mathrm{~s}, 6 \mathrm{H}, 2 \times \mathrm{CH}_{3}\right) ;{ }^{13} \mathrm{C} \mathrm{NMR}$ $\left(125 \mathrm{MHz}, \mathrm{CDCl}_{3}\right) \delta 169.1,168.1,136.5,133.4,132.4,131.9$, 129.0, 124.6, 124.4 121.0, 117.6, 42.7, 40.0; HRMS: found (ESI): $[\mathrm{M}+\mathrm{H}]^{+} 258.1256 \mathrm{C}_{14} \mathrm{H}_{16} \mathrm{~N}_{3} \mathrm{O}_{2}$, requires 258.1237.

2-Benzyl-4-((2,2-dimethylhydrazono)methyl)isoindoline-1,3dione $3 \mathrm{~m}$. A mixture of hydrazone $1 \mathrm{a}(100 \mathrm{mg}, 0.70 \mathrm{mmol}, 1.0$ eq.) and $N$-benzylmaleimide (198 mg, $1.05 \mathrm{mmol}, 1.5$ eq.) in [bmim][Cl] (1.0015 g) was heated at $100{ }^{\circ} \mathrm{C}$ by microwave irradiation for $2 \mathrm{~h}$. Water $(10 \mathrm{~mL})$ was added to the reaction mixture and the resulting mixture was extracted with EtOAc $(4 \times 10 \mathrm{~mL})$. The combined organic phase was washed with brine, dried over
$\mathrm{Na}_{2} \mathrm{SO}_{4}$ and filtered. The filtrate was concentrated to give a crude product which was recrystallised from water-acetone to give 5c as a yellow solid (208 $\mathrm{mg}$, 94\%). The remaining water phase was concentrated and dried to recover the ionic liquid (0.9633 g, 96\% recovery).

Yellow solid; mp $48-49{ }^{\circ} \mathrm{C} ;{ }^{1} \mathrm{H}$ NMR $\left(500 \mathrm{MHz}, \mathrm{CDCl}_{3}\right) \delta 8.22$ (d, $J=8.0 \mathrm{~Hz}, 1 \mathrm{H}, \mathrm{NNCHCCH}), 8.08$ (s, 1H, NNCH), 7.61 (d, $J=$ $7.0 \mathrm{~Hz}, 1 \mathrm{H}, \mathrm{NNCHCCHCHCH}), 7.55(\mathrm{t}, J=7.7 \mathrm{~Hz}, 1 \mathrm{H}$, $\mathrm{NNCHCCHCH}), 7.42$ (d, $J=7.3 \mathrm{~Hz}, 2 \mathrm{H}, \mathrm{Ph}), 7.35-7.22(\mathrm{~m}, 3 \mathrm{H}$, $\mathrm{Ph}), 4.82$ (s, 2H, $\mathrm{PhCH}_{2}$ ), $3.11\left(\mathrm{~s}, 6 \mathrm{H}, 2 \times \mathrm{CH}_{3}\right) ;{ }^{13} \mathrm{C}$ NMR (125 $\left.\mathrm{MHz}, \mathrm{CDCl}_{3}\right) \delta 169.2,168.3,136.7,136.5,133.4,132.4,129.1$, 128.8, 128.6, 127.8, 124.7, 124.4, 121.0, 42.7, 41.5; HRMS: found (ESI): $[\mathrm{M}+\mathrm{H}]^{+} 308.14024 \mathrm{C}_{18} \mathrm{H}_{18} \mathrm{~N}_{3} \mathrm{O}_{2}$, requires 308.13990; data in agreement with the literature. ${ }^{4}$

4-((2,2-Dimethylhydrazono)methyl)-2-(4-methoxybenzyl)isoindoline-1,3-dione 3n. A mixture of hydrazone $1 \mathrm{a}(100 \mathrm{mg}$, $0.72 \mathrm{mmol}$ ) and $\mathrm{N}$-4-methoxybenzylmaleimide (230 mg, 1.05 $\mathrm{mmol})$ in [bmim][Cl] $(0.9541 \mathrm{~g})$ was heated at $100{ }^{\circ} \mathrm{C}$ by microwave irradiation for $2 \mathrm{~h}$. Water $(20 \mathrm{~mL})$ was added and the reaction mixture was extracted with EtOAc $(5 \times 10 \mathrm{~mL})$. The combined organic extracts were washed with water, brine, dried $\left(\mathrm{Na}_{2} \mathrm{SO}_{4}\right)$, filtered, and concentrated in vacuo. The residue was recrystallised from acetone and cold water and the product collected by suction-filtration as a yellow solid (229 mg, 93\%). The remaining water phase was concentrated and dried to recover the ionic liquid ( $0.9395 \mathrm{~g}, 98 \%$ recovery).

Yellow solid; mp $129-131{ }^{\circ} \mathrm{C}$; $\nu_{\max }$ (solid per $\mathrm{cm}$ ) 2949, 2833, 1755, 1697, 1611, 1549, 1510; ${ }^{1} \mathrm{H}$ NMR $\left(500 \mathrm{MHz}, \mathrm{CDCl}_{3}\right) \delta 8.21$ (d, $J=8.0 \mathrm{~Hz}, 1 \mathrm{H}, \mathrm{NNCHCCH}), 8.08(\mathrm{~s}, 1 \mathrm{H}, \mathrm{NNCH}), 7.63-7.57$ $(\mathrm{m}, 1 \mathrm{H}, \mathrm{NNCHCCHCHCH}), 7.54(\mathrm{t}, J=7.6 \mathrm{~Hz}, 1 \mathrm{H}$, $\mathrm{NNCHCCHCH}), 7.37$ (d, $J=8.6 \mathrm{~Hz}, 2 \mathrm{H}, \mathrm{Ar}), 6.86-6.80(\mathrm{~m}, 2 \mathrm{H}$, $\mathrm{Ar}), 4.76\left(\mathrm{~s}, 2 \mathrm{H}, \mathrm{ArCH}_{2}\right), 3.76\left(\mathrm{~s}, 3 \mathrm{H}, \mathrm{OCH}_{3}\right), 3.11(\mathrm{~s}, 6 \mathrm{H}, 2 \times$ $\left.\mathrm{CH}_{3}\right) ;{ }^{13} \mathrm{C}$ NMR $\left(125 \mathrm{MHz}, \mathrm{CDCl}_{3}\right) \delta 169.2,168.3,159.2,136.5$, 133.4, 132.4, 130.1, 129.0, 128.9, 124.7, 124.5, 121.0, 114.1, 55.4, 42.7, 41.0; HRMS: found (ESI): $[\mathrm{M}+\mathrm{H}]^{+} 338.15041 \mathrm{C}_{19} \mathrm{H}_{20} \mathrm{~N}_{3} \mathrm{O}_{3}$, requires 338.15047 .

4-((2,2-Dimethylhydrazono)methyl)-2-phenethylisoindoline1,3-dione 3o. A mixture of hydrazone $1 \mathrm{a}(100 \mathrm{mg}, 0.72 \mathrm{mmol})$ and $N$-phenethylmaleimide (145 mg, $1.05 \mathrm{mmol})$ in [bmim] [Cl] $(1.0348 \mathrm{~g})$ was heated at $100^{\circ} \mathrm{C}$ by microwave irradiation for $2 \mathrm{~h}$. Water $(20 \mathrm{~mL})$ was added and the reaction mixture was extracted with EtOAc $(3 \times 20 \mathrm{~mL})$. The combined organic extracts were washed with water, brine, dried $\left(\mathrm{Na}_{2} \mathrm{SO}_{4}\right)$, filtered, and concentrated in vacuo. The residue was recrystallised from acetone and cold water and the product collected by suctionfiltration as a yellow solid (208 $\mathrm{mg}, 89 \%)$. The remaining water phase was concentrated and dried to recover the ionic liquid (1.0327 g, >99\% recovery).

Yellow solid; mp $106-107^{\circ} \mathrm{C} ; \nu_{\max }$ (solid per cm) 2931, 2864, 1763, 1694, 1541, 1454; ${ }^{1} \mathrm{H}$ NMR (500 MHz, $\left.\mathrm{CDCl}_{3}\right) \delta 8.22$ (d, $J=$ $7.9 \mathrm{~Hz}, 1 \mathrm{H}, \mathrm{Ar}), 8.09$ (s, 1H, NCH), $7.62-7.58$ (m, 1H, Ar), 7.55 (t, $J$ $=7.6 \mathrm{~Hz}, 1 \mathrm{H}, \mathrm{Ar}), 7.31-7.25(\mathrm{~m}, 4 \mathrm{H}, \mathrm{Ph}), 7.24-7.19(\mathrm{~m}, 1 \mathrm{H}, \mathrm{Ph})$, 3.94-3.83 (m, $\left.2 \mathrm{H}, \mathrm{PhCH}_{2} \mathrm{CH}_{2}\right), 3.12\left(\mathrm{~s}, 6 \mathrm{H}, 2 \times \mathrm{CH}_{3}\right), 3.02-2.92$ $\left(\mathrm{m}, 2 \mathrm{H}, \mathrm{PhCH}_{2}\right) ;{ }^{13} \mathrm{C}$ NMR $\left(125 \mathrm{MHz}, \mathrm{CDCl}_{3}\right) \delta$ 169.4, 168.3, 138.2, 136.4, 133.4, 132.4, 129.0, 128.9, 128.7, 126.7, 124.7, 124.5, 120.9, 42.7, 39.3, 34.9; HRMS: found (ESI): $[\mathrm{M}+\mathrm{H}]^{+}$ 322.15728 $\mathrm{C}_{19} \mathrm{H}_{20} \mathrm{~N}_{3} \mathrm{O}_{2}$, requires 322.15555. 
4-((2,2-Dimethylhydrazono)methyl)-2-ethyl-7methylisoindoline-1,3-dione 3p. A mixture of hydrazone $\mathbf{1 b}$ (111 mg, $0.73 \mathrm{mmol}$ ) and $N$-ethylmaleimide (139 mg, 1.11 $\mathrm{mmol})$ in $[\mathrm{bmim}][\mathrm{Cl}](1.0145 \mathrm{~g})$ was heated at $100{ }^{\circ} \mathrm{C}$ by microwave irradiation for $2 \mathrm{~h}$. Water $(20 \mathrm{~mL})$ was added and the reaction mixture was extracted with EtOAc $(5 \times 10 \mathrm{~mL})$. The combined organic extracts were washed with water, brine, dried $\left(\mathrm{Na}_{2} \mathrm{SO}_{4}\right)$, filtered, and concentrated in vacuo. The residue was recrystallised from acetone and cold water and the product collected by suction-filtration as a yellow solid (119 mg, 65\%). The remaining water phase was concentrated and dried to recover the ionic liquid (1.0013 $\mathrm{g}$, 98\% recovery).

Yellow solid; mp 142-143 ${ }^{\circ} \mathrm{C}$; ${ }^{1} \mathrm{H}$ NMR (500 MHz, $\mathrm{CDCl}_{3}$ ) $\delta 8.14(\mathrm{~s}, 1 \mathrm{H}, \mathrm{NNCH}), 8.08$ (d, $J=8.3 \mathrm{~Hz}, 1 \mathrm{H}, \mathrm{NNCHCCH}), 7.30$ (d, $J=8.3 \mathrm{~Hz}, 1 \mathrm{H}, \mathrm{NNCHCCHCH}), 3.68(\mathrm{~d}, J=7.1 \mathrm{~Hz}, 2 \mathrm{H}$, $\left.\mathrm{CH}_{2} \mathrm{CH}_{3}\right), 3.08\left(\mathrm{~s}, 6 \mathrm{H}, 2 \times \mathrm{CH}_{3}\right), 2.63\left(\mathrm{~s}, 3 \mathrm{H}, \mathrm{CH}_{3}\right), 1.24(\mathrm{t}, J=$ $\left.7.1 \mathrm{~Hz}, 3 \mathrm{H}, \mathrm{CH}_{2} \mathrm{CH}_{3}\right) ;{ }^{13} \mathrm{C} \mathrm{NMR}\left(125 \mathrm{MHz}, \mathrm{CDCl}_{3}\right) \delta 169.3,169.1$, 136.2, 135.8, 134.1, 128.8, 128.7, 125.6, 125.2, 42.7, 32.6, 17.6, 14.1; HRMS: found (ESI): $[\mathrm{M}+\mathrm{H}]^{+} 260.1398 \mathrm{C}_{14} \mathrm{H}_{18} \mathrm{~N}_{3} \mathrm{O}_{3}$, requires 260.1399; data in agreement with the literature. ${ }^{4}$

4-((2,2-Dimethylhydrazono)methyl)-7-methyl-2-

phenylisoindoline-1,3-dione 3q. A mixture of hydrazone $\mathbf{1 b}$ (119 mg, $0.78 \mathrm{mmol}$ ) and $N$-phenylmaleimide (197 mg, 1.14 $\mathrm{mmol})$ in $[\mathrm{bmim}][\mathrm{Cl}](0.9931 \mathrm{~g})$ was heated at $100{ }^{\circ} \mathrm{C}$ by microwave irradiation for $2 \mathrm{~h}$. Water $(20 \mathrm{~mL})$ was added and the reaction mixture was extracted with EtOAc $(5 \times 10 \mathrm{~mL})$. The combined organic extracts were washed with water, brine, dried $\left(\mathrm{Na}_{2} \mathrm{SO}_{4}\right)$, filtered, and concentrated in vacuo. The residue was recrystallised from acetone and cold water and the product collected by suction-filtration as a yellow solid (109 mg, 46\%). The remaining water phase was concentrated and dried to recover the ionic liquid ( $0.9893 \mathrm{~g}$, 99\% recovery).

Yellow solid; mp 201-203 ${ }^{\circ} \mathrm{C}$; $\nu_{\max }$ (solid per cm) 1753, 1694, 1541, 1489; ${ }^{1} \mathrm{H}$ NMR (500 $\left.\mathrm{MHz}, \mathrm{CDCl}_{3}\right) \delta 8.18-8.17(\mathrm{~m}, 2 \mathrm{H}$, $\mathrm{NNCH}$ and $\mathrm{NNCHCCH}), 7.49(\mathrm{t}, J=7.8 \mathrm{~Hz}, 2 \mathrm{H}, \mathrm{Ph}), 7.45-7.36$ $(\mathrm{m}, 4 \mathrm{H}, \mathrm{Ph}$ and NNCHCCHCH$), 3.09\left(\mathrm{~s}, 6 \mathrm{H}, 2 \times \mathrm{CH}_{3}\right), 2.69(\mathrm{~s}$, $\left.3 \mathrm{H}, \mathrm{CH}_{3}\right) ;{ }^{13} \mathrm{C}$ NMR $\left(125 \mathrm{MHz}, \mathrm{CDCl}_{3}\right) \delta 168.4,168.1,136.7$, 136.4 , 134.7, 131.9, 129.2, 129.1, 128.2, 128.0, 126.9, 125.3, 124.5, 42.7, 17.8; HRMS: found (ESI): $[\mathrm{M}+\mathrm{H}]^{+} 308.1341$ $\mathrm{C}_{18} \mathrm{H}_{28} \mathrm{~N}_{3} \mathrm{O}_{3}$, requires; 308.1399 .

4-((2,2-Dimethylhydrazono)methyl)-2-hexyl-7-

methylisoindoline-1,3-dione 3r. A mixture of hydrazone $\mathbf{1 b}$ ( $80 \mathrm{mg}, 0.69 \mathrm{mmol}$ ) and $N$-hexylmaleimide (154 mg, $0.84 \mathrm{mmol}$ ) in [bmim] $[\mathrm{Cl}](1.00 \mathrm{~g})$ was heated at $100{ }^{\circ} \mathrm{C}$ by microwave irradiation for $2 \mathrm{~h}$. Water $(20 \mathrm{~mL})$ was added and the reaction mixture was extracted with EtOAc $(5 \times 10 \mathrm{~mL})$. The combined organic extracts were washed with water, brine, dried $\left(\mathrm{Na}_{2} \mathrm{SO}_{4}\right)$, filtered, and concentrated in vacuo. The residue was recrystallised from acetone and cold water and the product collected by suction-filtration as a yellow solid (110 mg, 67\%). The remaining water phase was concentrated and dried to recover the ionic liquid ( $0.97 \mathrm{~g}, 97 \%$ recovery).

Yellow solid; $\mathrm{mp} 80-81{ }^{\circ} \mathrm{C} ; \nu_{\max }$ (solid per cm) 2929, 1693, 1548; ${ }^{1} \mathrm{H}$ NMR $\left(500 \mathrm{MHz}, \mathrm{CDCl}_{3}\right) \delta 8.13(\mathrm{~s}, 1 \mathrm{H}, \mathrm{NNCH}), 8.07$ (d, $J$ $=8.2 \mathrm{~Hz}, 1 \mathrm{H}, \mathrm{NNCHCCH}), 7.29(\mathrm{~d}, J=8.2 \mathrm{~Hz}, 1 \mathrm{H}$, $\mathrm{NNCHCCHCH}), 3.64-3.56\left(\mathrm{~m}, 2 \mathrm{H}, \mathrm{NCH}_{2}\left(\mathrm{CH}_{2}\right)_{4} \mathrm{CH}_{3}\right), 3.07$ (s, $6 \mathrm{H}$, $\left.2 \times \mathrm{CH}_{3}\right), 2.63\left(\mathrm{~s}, 3 \mathrm{H}, \mathrm{CH}_{3}\right), 1.66-1.60\left(\mathrm{~m}, 2 \mathrm{H}, \mathrm{CH}_{2}\right), 1.34-1.28$ $\left(\mathrm{m}, 6 \mathrm{H}, 3 \times \mathrm{CH}_{2}\right), 0.86\left(\mathrm{t}, J=6.9 \mathrm{~Hz}, 3 \mathrm{H}, \mathrm{NCH}_{2}\left(\mathrm{CH}_{2}\right)_{4} \mathrm{CH}_{3}\right) ;{ }^{13} \mathrm{C}$ $\mathrm{NMR}\left(125 \mathrm{MHz}, \mathrm{CDCl}_{3}\right) \delta 169.5,169.3,136.2,135.8,134.0,128.7$, 128.6, 125.6, 125.1, 42.7, 37.8, 31.5, 28.7, 26.7, 22.6, 17.6, 14.1; HRMS: found (ESI): $[\mathrm{M}+\mathrm{H}]^{+} 316.20474 \mathrm{C}_{18} \mathrm{H}_{26} \mathrm{~N}_{3} \mathrm{O}_{2}$, requires 316.20250.

\section{4-((2,2-Dimethylhydrazono)methyl)-2-ethyl-7-}

hydroxyisoindoline-1,3-dione 3s. A mixture of hydrazone 1c (72 $\mathrm{mg}, 0.35 \mathrm{mmol}$ ) and $N$-ethylmaleimide $(67 \mathrm{mg}, 0.53 \mathrm{mmol})$ in $[\mathrm{bmim}][\mathrm{Cl}](1.595 \mathrm{~g})$ was heated at $100{ }^{\circ} \mathrm{C}$ by microwave irradiation for $2 \mathrm{~h}$. Water $(20 \mathrm{~mL})$ was added and the reaction mixture was extracted with EtOAc $(5 \times 20 \mathrm{~mL})$. The combined organic extracts were washed with water, brine, dried $\left(\mathrm{Na}_{2} \mathrm{SO}_{4}\right)$, filtered, and concentrated in vacuo. The residue was recrystallised from acetone and cold water and the product collected by suction-filtration as a red-brown solid (58 $\mathrm{mg}, 63 \%)$. The remaining water phase was concentrated and dried to recover the ionic liquid $(1.67 \mathrm{~g},>100 \%$ recovery, partial contamination with product). Data in agreement with the literature. ${ }^{4}$

Red-brown solid; mp 147-148 ${ }^{\circ} \mathrm{C} ;{ }^{1} \mathrm{H}$ NMR (500 MHz, $\mathrm{CDCl}_{3}$ ) $\delta 8.15(\mathrm{~d}, J=8.7 \mathrm{~Hz}, 1 \mathrm{H}, \mathrm{NNCHCCH}), 8.00(\mathrm{~s}, 1 \mathrm{H}, \mathrm{NNCH}), 7.73$ (br s, 1H, OH), 7.07 (d, $J=8.7 \mathrm{~Hz}, 1 \mathrm{H}, \mathrm{NNCHCCHCH}), 3.69$ (q, $J$ $\left.=7.3 \mathrm{~Hz}, 2 \mathrm{H}, \mathrm{CH}_{2}\right), 3.07\left(\mathrm{~s}, 6 \mathrm{H}, 2 \times \mathrm{CH}_{3}\right), 1.27(\mathrm{t}, J=7.3 \mathrm{~Hz}, 3 \mathrm{H}$, $\left.\mathrm{CH}_{3}\right) ;{ }^{13} \mathrm{C}$ NMR $\left(125 \mathrm{MHz}, \mathrm{CDCl}_{3}\right) \delta 170.5,168.6,153.7,132.4$, 129.8, 125.3, 124.2 123.1, 113.5, 42.8, 32.7, 14.2; HRMS: found (ESI): $[\mathrm{M}+\mathrm{Na}]^{+} 284.1018 \mathrm{C}_{13} \mathrm{H}_{15} \mathrm{~N}_{3} \mathrm{NaO}_{3}$, requires 284.1011.

4-((2,2-Dimethylhydrazono)methyl)-7-hydroxy-2-

phenylisoindoline-1,3-dione 3t. A mixture of hydrazone 1c (50 mg, $0.25 \mathrm{mmol}$ ) and $N$-phenylmaleimide (52 $\mathrm{mg}, 0.30 \mathrm{mmol}$ ) in $[\mathrm{bmim}][\mathrm{Cl}](1.869 \mathrm{~g})$ was heated at $100{ }^{\circ} \mathrm{C}$ by microwave irradiation for $2 \mathrm{~h}$. Water $(20 \mathrm{~mL})$ was added and the reaction mixture was extracted with EtOAc $(5 \times 20 \mathrm{~mL})$. The combined organic extracts were washed with water, brine, dried $\left(\mathrm{Na}_{2} \mathrm{SO}_{4}\right)$, filtered, and concentrated in vacuo. The residue was recrystallised from acetone and cold water and the product collected by suction-filtration as a red-brown solid (29 $\mathrm{mg}, 39 \%$ ). The remaining water phase was concentrated and dried to recover the ionic liquid (1.860 g, 99\%).

Red-brown solid; mp 227-229 ${ }^{\circ} \mathrm{C} ; \nu_{\max }\left(\mathrm{KBr} / \mathrm{cm}^{-1}\right) 3246$, 1763, 1686, 1557, 1493; ${ }^{1} \mathrm{H}$ NMR (500 MHz, $\left.\mathrm{CDCl}_{3}\right) \delta 8.24$ (d, $J=$ $8.8 \mathrm{~Hz}, 1 \mathrm{H}, \mathrm{NNCHCCH}), 8.03(\mathrm{~s}, 1 \mathrm{H}, \mathrm{NNCH}), 7.88(\mathrm{~s}, 1 \mathrm{H}, \mathrm{OH})$, 7.49 (d, $J=7.8 \mathrm{~Hz}, 2 \mathrm{H}, \mathrm{Ph}), 7.46-7.34$ (m, 3H, Ph), 7.15 (d, $J=$ $8.8 \mathrm{~Hz}, 1 \mathrm{H}, \mathrm{NNCHCCHCH}), 3.07\left(\mathrm{~s}, 6 \mathrm{H}, 2 \times \mathrm{CH}_{3}\right) ;{ }^{13} \mathrm{C} \mathrm{NMR}(125$ $\left.\mathrm{MHz}, \mathrm{CDCl}_{3}\right) \delta 169.5,167.6,154.2,132.9,131.4,130.3,129.2$, 128.2, 126.5, 124.9, 123.6, 123.2, 113.0, 42.76; HRMS: found (ESI): $[\mathrm{M}+\mathrm{H}]^{+} 301.1184 \mathrm{C}_{17} \mathrm{H}_{16} \mathrm{~N}_{3} \mathrm{O}_{2}$, requires 310.1192.

Recycling [bmim][Cl] in the synthesis of phthalimide $3 \mathrm{~b}$. A mixture of hydrazone $3 \mathrm{a}$ (1.0 eq.) and $N$-ethylmaleimide (1.5 eq.) in $[\mathrm{bmim}][\mathrm{Cl}]$ (8.2 eq.) was heated at $100{ }^{\circ} \mathrm{C}$ by microwave irradiation for $2 \mathrm{~h}$. Water $(20 \mathrm{~mL})$ was added and the reaction mixture was extracted with EtOAc $(3 \times 15 \mathrm{~mL})$. The combined organic extracts were washed with water, brine, dried $\left(\mathrm{Na}_{2} \mathrm{SO}_{4}\right)$, filtered, and concentrated in vacuo. The residue was further recrystallised from acetone with cold water and the product collected by suction-filtration as a yellow solid. The remaining water phase was concentrated to obtain recovered ionic liquid, which was then dissolved in $\mathrm{CH}_{2} \mathrm{Cl}_{2}$ and dried over $\mathrm{Na}_{2} \mathrm{SO}_{4}$. 
Filtration and concentration of the filtrate gave the recovered ionic liquid, which was used for the synthesis of $\mathbf{3 b}$ again. The same procedure was repeated five times.

\section{Conflicts of interest}

There are no conflicts to declare.

\section{Acknowledgements}

This work was partially supported by KAKENHI for Challenging Exploratory Research 17K19139. The authors gratefully acknowledge JSPS for doctoral fellowship funding provided to V. K. (PE17018).

\section{Notes and references}

1 A. Kamimura, T. Okagawa, N. Oyama, T. Otsuka and M. Yoshimoto, Green Chem., 2012, 14, 2816-2820.

2 N. Shi, Q. Liu, T. Wang, L. Ma, Q. Zhang and Q. Zhang, ACS Sustainable Chem. Eng., 2014, 2, 637-642.

3 J. Cui, J. Tan, T. Deng, X. Cui, Y. Zhu and Y. Li, Green Chem., 2016, 18, 1619-1624.

4 S. Higson, F. Subrizi, T. D. Sheppard and H. C. Hailes, Green Chem., 2016, 18, 1855-1858.

5 A. E. Settle, L. Berstis, N. A. Rorrer, Y. Roman-Leshkóv, G. T. Beckham, R. M. Richards and D. R. Vardon, Green Chem., 2017, 19, 3468-3492.

6 E. Ramírez, R. Bringué, C. Fité, M. Iborra, J. Tejero and F. Cunill, J. Chem. Technol. Biotechnol., 2017, 92, 2775-2786.

7 G. W. Huber, S. Iborra and A. Corma, Chem. Rev., 2006, 106, 4044-4098.

8 S. A. Sanchez-Vazquez, H. C. Hailes and J. R. G. Evans, Polym. Rev., 2013, 53, 627-694.

9 S. Shylesh, A. A. Gokhale, C. R. Ho and A. T. Bell, Acc. Chem. Res., 2017, 50, 2589-2597.

10 J. M. Fraile, J. I. García, M. A. Gomez, A. de la Hoz, J. A. Mayoral, A. Moreno, P. Prieto, L. Salvatella and E. Vázquez, Eur. J. Org. Chem., 2001, 2891-2899.

11 G. V. Kumar and A. Rajendran, Synth. Commun., 2016, 46, 483-496.

12 B. H. Shanks and P. L. Keeling, Green Chem., 2017, 19, 31773185.

13 J. J. Lee and G. A. Kraus, Green Chem., 2014, 16, 2111-2116. 14 E. Mahmoud, J. Yu, R. J. Gorte and R. F. Lobo, ACS Catal., 2015, 5, 6946-6955.
15 R. J. Van Putten, J. C. Van Der Waal, E. De Jong, C. B. Rasrendra, H. J. Heeres and J. G. De Vries, Chem. Rev., 2013, 113, 1499-1597.

16 R. Sheldon, Chem. Commun., 2001, 2399-2407.

17 R. Fu, Y. Yang, W. Feng, Q. Ge, Y. Feng, X. Zeng, W. Chai, J. Yi and R. Yuan, Tetrahedron, 2016, 72, 8319-8326.

18 B. Yuan, J. Guan, J. Peng, G. Zhou Zhu and J. Hong Jiang, Chem. Eng. J., 2017, 330, 109-119.

19 R. Ratti, Adv. Chem., 2014, 2014, 1-16.

20 T. Welton, Chem. Rev., 1999, 99, 2071-2084.

21 E. Dresler, E. Jasinska, A. Kryger-Lapczuk, E. NowakowskaBogdan and R. Jasinski, Chemik, 2015, 69, 288-296.

22 R. Martínez-Palou, Mol. Diversity, 2010, 14, 3.

23 E. Van der Eycken, P. Appukkuttan, W. De Borggraeve, W. Dehaen, D. Dallinger and C. O. Kappe, J. Org. Chem., 2002, 67, 7904.

24 J. Hoffmann, M. Nuchter, B. Ondruschka and P. Wasserscheid, Green Chem., 2003, 5, 296.

25 M. M. Dharman, H.-Y. Ju, H.-L. Shim, M.-K. Lee, K.-H. Kim and D.-W. Park, J. Mol. Catal. A: Chem., 2009, 303, 91.

26 N. E. Leadbeater and H. M. Torenius, J. Org. Chem., 2002, 67, 3145 .

27 K. S. A. Vallin, P. Emilsson, M. Larhed and A. Hallberg, J. Org. Chem., 2002, 67, 6243.

28 N. Z. Kiss and G. Keglevich, Tetrahedron Lett., 2016, 57, 971.

29 J. Tharun, A. C. Kathalikkattil, R. Roshan, D.-H. Kang, H.-C. Woo and D.-W. Park, Catal. Commun., 2014, 54, 31.

30 Y. Chen, Y. Zu, Y. Fu, X. Zhang, P. Yu, G. Sun and T. Efferth, Molecules, 2010, 15, 9486.

31 A. Kamimura and S. Yamamoto, Org. Lett., 2007, 9, 25322535.

32 A. Kamimura, S. Yamamoto and K. Yamada, ChemSusChem, 2011, 4, 644-649.

33 A. Kamimura, T. Okagawa, N. Oyama, T. Otsuka and M. Yoshimoto, Green Chem., 2012, 14, 2816-2820.

34 A. Kamimura, K. Murata, Y. Tanaka, T. Okagawa, H. Matsumoto, K. Kaiso and M. Yoshimoto, ChemSusChem, 2014, 7, 3257-3259.

35 D. R. MacFarlane, M. Forsyth, E. I. Izgorodina, A. P. Abbott, G. Annata and K. Fraser, Phys. Chem. Chem. Phys., 2009, 11, 4962-4967.

36 K. Ueno, H. Tokuda and M. Watanabe, Phys. Chem. Chem. Phys., 2010, 21, 1649-1658. 